\title{
Scientists rethink approach to HIV gels
}

Scientists and activists struggling to combat the HIV epidemic were shattered by last month's failure of a candidate microbicide gel in large clinical trials. Microbicides aim to block HIV infection, but the candidate product - cellulose sulphate - seemed to increase women's susceptibility. In the wake of this setback, some are now warning that the field must change its approach.

Dozens of candidate microbicides are being tested in labs around the world. But advocates of change say the field must make hard choices about which of these should graduate to phase III clinical trials - extended trials to confirm that a product works - because these trials are expensive and time-consuming. Such choices have not previously been made: the gels tested so far were the first to be ready, not necessarily the most promising.

"How many more clinical trials are we going to be able to do with dramatic failures like this?" asks Ronald Veazey, chair of the division of comparative pathology at the Tulane National Primate Research Center in Covington, Louisiana.

The microbicides tested in phase III trials so far all belong to a 'first generation' of products that aim to make the vagina less hospitable to HIV, but don't target the virus directly. So far, none of these has worked. One, Savvy gel, failed, and two - cellulose sulphate and the spermicide nonoxynol-9 - led to higher HIV infection rates. Phase III trials of three other products are ongoing, a redundancy that is now unavoidable as the trials have already begun.

Meanwhile, most researchers see more promise in a batch of microbicides at an earlier stage of development. These products contain gel formulations of antiretroviral drugs that target HIV. But for these microbicides, too, there are many similar products in the pipeline.

For instance, different groups are testing at least four microbicide candidates belonging to a class of drug known as non-nucleoside reverse transcriptase inhibitors (NNRTIs). Two of these will probably be ready for phase III trials before 2009. The question now is whether all the products should automatically be pushed into such trials.

"Are we really going to proceed with two different NNRTIs, or should we be doing a study comparing them head-to-head, to help us determine which move forward?" says Salim Abdool Karim, director of an AIDS research centre at the University of KwaZulu-Natal in Durban, South Africa.

Right now, it is difficult to choose the best

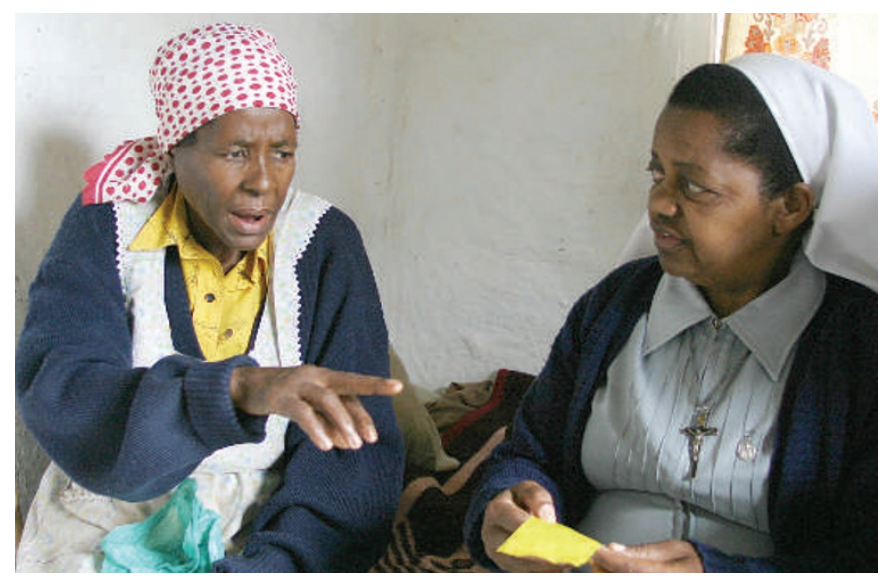

The recent failure of clinical trials of a microbicidal gel has highlighted the need to make tough decisions. candidates without doing large human studies because different research groups use different preclinical testing methods, and there is no agreement on which of these tests best predict how a microbicide will act in patients.

The failure of cellulose sulphate may actually help solve this problem. CONRAD, the Virginia-based agency that organized trials of cellulose sulphate in five countries, is determined to find out why the compound was harmful. "We'll probably put together a blinded panel of compounds, including cellulose sulphate, and send it out to various individuals working on different models and see if they can come up with any clues," says Henry Gabelnick, CONRAD’s executive director. Testing the same products across different models could help researchers compare different microbicides in future.

But that will leave a bigger obstacle: each research group is investing in its own product, so will be reluctant to step aside for others. The field may need a guiding hand from an impar- tial body, which doesn't exist at the moment.

It has been suggested that a working group on microbicides currently being set up by the US National Institutes of Health's Office of AIDS Research may serve this role. Its composition and role have not been clearly defined, but advocates suggest it could solicit advice from independent experts about which products should proceed to clinical trials.

Some, including Gabelnick, say it would be a mistake to jettison products just to avoid redundancy. "We don't know enough to say these are all the same," he argues.

But others, such as Polly Harrison, director of the Alliance for Microbicide Development based in Silver Spring, Maryland, say someone has to help the field reach a consensus. "Prioritization has to occur, and it has to go all the way along the pipeline," Harrison says. "We know we need this."

Erika Check

See Editorial, page 1.

\section{Trio of studies makes headway in HIV battle}

The search for new ways to curb HIV transmission received a badly needed boost last week.

On 22 February, researchers reported that drug treatment against the herpes simplex 2 virus cuts levels of HIV RNA in the blood and genitals of women infected with both viruses (N. Nagot et al. N. Engl. J. Med. 356, 790-799; 2007). The researchers suggest that controlling herpes may thus also control the spread of HIV. Studies are under way to test the idea. The following day, two large controlled trials on male circumcision in Uganda and Kenya were published (M. L. Newell \& T. Bärnighausen Lancet 369, 617-619; 2007). Both trials had been stopped early by their funder, the US National Institutes of Health, because the effects of the procedure were already clear. Together with a previous study in South Africa, the results show that circumcision can reduce a man's risk of HIV infection by 50-60\%.

The task now is to roll out circumcision in countries that would most benefit, where HIV rates are high and the virus is spread mainly through heterosexual sex. A working group convened by the Joint United Nations Programme on AIDS and the World Health Organization will hold a consultation on 6 March to discuss how and where circumcision should be provided.
Erika Check 